\title{
Planungsintegrierter Einsatz der Simulationstechnik im Investitionsprozess eines Produktionskomplexes
}

\author{
Astrid Oberschmidt, Dieter Hartrampf, Claus-Gerold Grundig
}

Die Verfasser bedanken sich bei Herrn Dr.-Ing. Batge, Geschäftsführer der SMB Innovative Produkte Wildau GmbH, und Herrn Dipl.-Ing. Soost, Bereich Entwicklung/Fertigung, für die konsequent durchgängige Projektzusammenarbeit.

\section{Problemstellung}

Ein mittelständiges Unternehmen des Maschinenbaus stand vor der Investitionsaufgabe einen Produktionskomplex zur hochrationellen Produktion von innovativen Kleinteilen im Mittel- und Großserienbereich neu zu bauen.

Zur Unterstuitzung und Qualifizierung des erforderlichen Planungs- und Entscheidungsprozesses dieser komplexen Fabrikplanungsaufgabe wurden durch das Unternehmen schon frühzeitig mit der Planungsaufnahme Analysekapazitäten des Labors „Fabrikplanung“ der TFH Wildau vertraglich gebunden und eingesetzt. Die über einen mittelfristigen Zeitraum zu realisierenden Projekte „Angewandter Forschung“" (Technologietransfer) beinhalteten den Einsatz von Simulationstools mit Zielsetzungen zur Abbildung und Analyse des zu planenden Investitionsobjektes zeitparallel integriert über den gesamtem Planungs-, Investitions- und Inbetriebnahmeablauf.

Im nachfolgenden Erfahrungsbericht werden die durchgängige, planungsbegleitende Realisierung wesentlicher Simulationskomplexe sowie ausgewählte Simulationsergebnisse der realisierten Projekte zusammenfassend vorgestellt. Für die fachwissenschaftliche Einordnung dieser Untersuchungen ist von Bedeutung:

- Der Einsatz von Simulationstechniken zur Qualifizierung der Planungsprozesse von Fabrik- und Produktionssystemen ist weitgehend Standard in Großunternehmen und Forschungsinstitutionen bzw. wird als Dienstleistung in großer Breite angeboten (Engineering-Center).

- Deutlich defizitär dagegen ist der Breiteneinsatz der Simulationstechnik noch heute in klein- und mittelständigen Unternehmen (KMU). Verbreitete Ursachen sind Kenntnis- und Erfahrungsmängel in den Unternehmen (vgl. Aussagen und Analysen z. B. in [1] bis [6]).

- Erfordernisse und Erfahrungen zur methodischen Integration von Simulationsexperimenten in den Planungsablauf sind in der Fachliteratur dominant auf punktuelle Einsatzszenarien begrenzt.

Wesentliches Anliegen der realisierten Simulationsprojekte war es daher, Erfahrungen und Probleme beim
Einsatz der Simulationstechnik unter den spezifischen Bedingungen von KMU aufzuzeigen und dabei insbesondere die Notwendigkeit des ,planungsbegleitenden Einsatzes" deutlich zu machen. Absicht entsprechender Untersuchungen sollte es u. a. auch sein, die vorhandenen Akzeptanz- bzw. Anwendungsdefizite in KMU durch die Entwicklung „überzeugender Beispiellösungen“ (Musterprojekte) abzubauen (vgl. z. B. Strategien in [6]).

\section{Simulationstechnik im Planungs- und Investitionsprozess}

In der industriellen Praxis wird die Methodik des komplexen Investitionsplanungsprozesses von Fabrik- und Produktionssystemen maßgeblich durch generalisierte Planungs- und Entscheidungsetappen des strukturierten Fabrikplanungsprozesses (Fabrikplanungssystematiken vgl. z. B. [7] bis [9]) charakterisiert. Dieser Planungs- und Projekterarbeitungsprozess umfasst mehrere, funktionell und ablaufbedingt inhaltlich unterschiedliche Planungsphasen steigender Planungsgenauigkeit . Deren zeitliche Abfolge bzw. Ausdehnung ist zur Sicherung von Wirtschaftlichkeits- und Nutzungsparametern des Planungsobjektes prinzipiell kurz zu halten.

Im industriellen Planungsfall unterliegt die reale zeitliche Ablaufstruktur des Planungsprozesses allerdings dominant dem jeweiligen Kenntnis- bzw. Entscheidungsstand der Unternehmensleitung. Dieser steht in der Folge wechselnder Sachzwänge und oftmals unscharfer Planungsdaten (Dynamik von Markt-, Finanz- und Preisentwicklungen), so dass Zeitabläufe und Terminentwicklungen zum Investitionsobjekt und damit zum Planungsablauf hierdurch maßgeblich bestimmt bzw. verzögert oder beschleunigt werden. Dieser in der industriellen Praxis hochdynamische, von Risiken und Unsicherheiten uiberlagerte Planungsablauf ist folglich charakterisiert durch

- zeitliche Abfolgen veränderter Vorgaben, Zielsetzungen, Szenarien, Entscheidungen,

- Eingriffe, Korrekturen, Überplanungen, Wiederholungen,

- Zeitverzögerungen, Überlappungen, Unterbrechungen und Beschleunigungen.

Damit wird deutlich, dass der Einsatz der Simulationstechnik im Planungsablauf nicht einmalig-punktuell, sondern planungs- bzw. projektbegleitend zyklisch, d. h. in den Planungsablauf integriert, durchzusetzen ist. Nur so können aktuelle Entwicklungen und Korrekturen erfasst sowie die Sicherung von Planungsergebnissen entsprechend der Planungssystematik durchgesetzt werden. 
Simulationsuntersuchungen sind folglich über den gesamten Planungsablauf, zeitlich versetzt, zyklisch wiederholend unter permanenter Modellanpassung mit Zielsetzungen planungsfortschreitend steigender Aussagequalität einzuordnen. Nur diese gezielte, durchgängige Integration der Simulationsuntersuchungen sichert dem jeweiligen Planungsstand entsprechende aktuelle Entscheidungshilfen.

Im vorliegenden Investitionsobjekt umfasste der gesamte Planungsablauf einen mehrjährigen Zeitraum. Der entsprechende Planungs-, Investitions- und Inbetriebnahmeprozess wurde in Abstimmung mit dem Auftraggeber durchgängig begleitet durch gezielt eingeordnete, aufeinander aufbauende Simulationskomplexe (Teilprojekte) bei jeweils veränderten Datenbasen, Problemstellungen und Zielsetzungen entsprechend dem jeweils erreichten Planungsstand.

\section{Planungsintegrierte Simulations- komplexe - Industriebeispiel}

\subsection{Merkmale der Simulationskomplexe}

Die Einordnung von Simulationsuntersuchungen in den Planungsprozess kann - wie in Abbildung 1 aufgezeigt - entsprechend den Planungsphasen der Fabrikplanungssystematik vorgenommen werden [10]. Der Einsatz kann folglich unterschieden werden z. B. in Untersuchungen in der Vor-, Grob- oder auch Feinplanung des Planungsobjektes. Entsprechend den jeweils erreichten Planungszeitpunkten und -inhalten sind die Problemstellungen, Umfang und Genauigkeit der Daten sowie der Detaillierungs- bzw. Abstraktionsgrad des Simulationsmodells deutlich unterschiedlich. Entsprechend der Logik der Fabrikplanungssystematik tritt mit Planungsfortgang ein deutlicher Anstieg der Daten-, Modell- und Ergebnisqualität ein. Eine gezielte Einordnung von Simulationstools ermöglicht folglich permanent Experimente zu speziellen Problem- stellungen innerhalb unterschiedlicher Planungsphasen, so z. B. in der

- Vorplanung zu erforderlichen Auslegungsgrößen und Investitionsbedarfen, in der

- Grobplanung zur Strukturierung, Optimierung, Funktionskontrolle und Variantenauswahl und in der

- Feinplanung zur detaillierten Feinauslegung und Anlaufplanung der Ausfuihrungsvariante.

Zeitlicher Abstand, Einordnung und Umfang der Experimente sind durch den Planungsfortschritt, die jeweilige Problemlage, den Umfang von Eingriffen und Korrekturen sowie die Erfordernisse der aktuellen Entscheidungssituation gegeben. Dabei sind zeitlich-inhaltliche Überlagerungen von Problemstellungen und Modellinhalten im realen Anwendungsfall möglich.

Wie in Tafel 1 dargestellt, wurden im vorliegenden industriellen Anwendungsfall die Untersuchungen in drei inhaltlich unterschiedliche Simulationskomplexe A, B und $C$ gegliedert. Dabei wurden die Komplexe B und $C$ auf Grund identischer Modellstrukturen integriert realisiert. Diese Simulationskomplexe wurden im Rahmen eigenständiger Teilprojekte in Abstimmung mit dem Auftraggeber zeitversetzt bearbeitet und umfassen jeweils eine Vielzahl spezieller statischer Berechnungen und darauf aufsetzender Simulationspakete (vgl. Projektdokumentationen in [13] bis [16]).

Die vom Auftraggeber vorgegebene komplexe Aufgabenstellung war wie folgt charakterisiert: Gesucht sind solche Systemkonfigurationen des Produktionskomplexes, bei denen Mindestdurchsatzgrößen (Umsatzvolumen) unterschiedlicher Szenarien (Produktionsprogramme) unter Einhaltung spezieller Restriktionen wirtschaftlich produziert werden können. Als wesentliche Restriktionen waren angesetzt

- Einhaltung von Auslastungsgrößen der Ausrüstungen (Auslastungsgrade, Schichtstrukturen),

- Begrenzung und Homogenisierung der Staubildung der Produkte (Lager- und Pufferabgleich).

\begin{tabular}{|l|l|l|}
\hline $\begin{array}{l}\text { Planungssystematik } \\
\text { Planungsphase }\end{array}$ & $\begin{array}{l}\text { Industrieprojekt } \\
\text { Simulationskomplexe }\end{array}$ & $\begin{array}{l}\text { Untersuchungsschwerpunkte } \\
\text { Simulationskomplex }\end{array}$ \\
\hline $\begin{array}{l}\text { Vorplanung } \\
\text { Entscheidungsgrundlage } \\
\text { Investitionsobjekt } \\
\text { (Pre feasibility studie) }\end{array}$ & $\begin{array}{l}\text { Simulationskomplex A } \\
\text { Teilprojekte [13], [14] }\end{array}$ & $\begin{array}{l}\text { Grobentwurf Lösungsprinzip } \\
\text { (Szenarien) } \\
\text { Grobdimensionierung - } \\
\text { Ausrüstungsumfang } \\
\text { Investitionsaufwände, Flächen- } \\
\text { und Raumbedarfe }\end{array}$ \\
\hline $\begin{array}{l}\text { Grobplanung } \\
\text { Entwurf Varianten / } \\
\text { Variantenauswahl } \\
\text { (feasibility studie) } \\
\text { Feinplanung } \\
\begin{array}{l}\text { Feinoptimierung - } \\
\text { Ausführungsvariante } \\
\text { (Ausführungsprojekt) }\end{array}\end{array}$ & $\begin{array}{l}\text { Simulationskomplex B } \\
\text { Teilprojekte [15], [16] }\end{array}$ & $\begin{array}{l}\text { Entwurf/Korrektur alternativer } \\
\text { Lösungsvarianten } \\
\text { Ableitung Vorzugsvarianten }\end{array}$ \\
\hline
\end{tabular}

Tafel 1: Definition der Simulationskomplexe A, B und C im Planungsablauf - Investitionsobjekt 


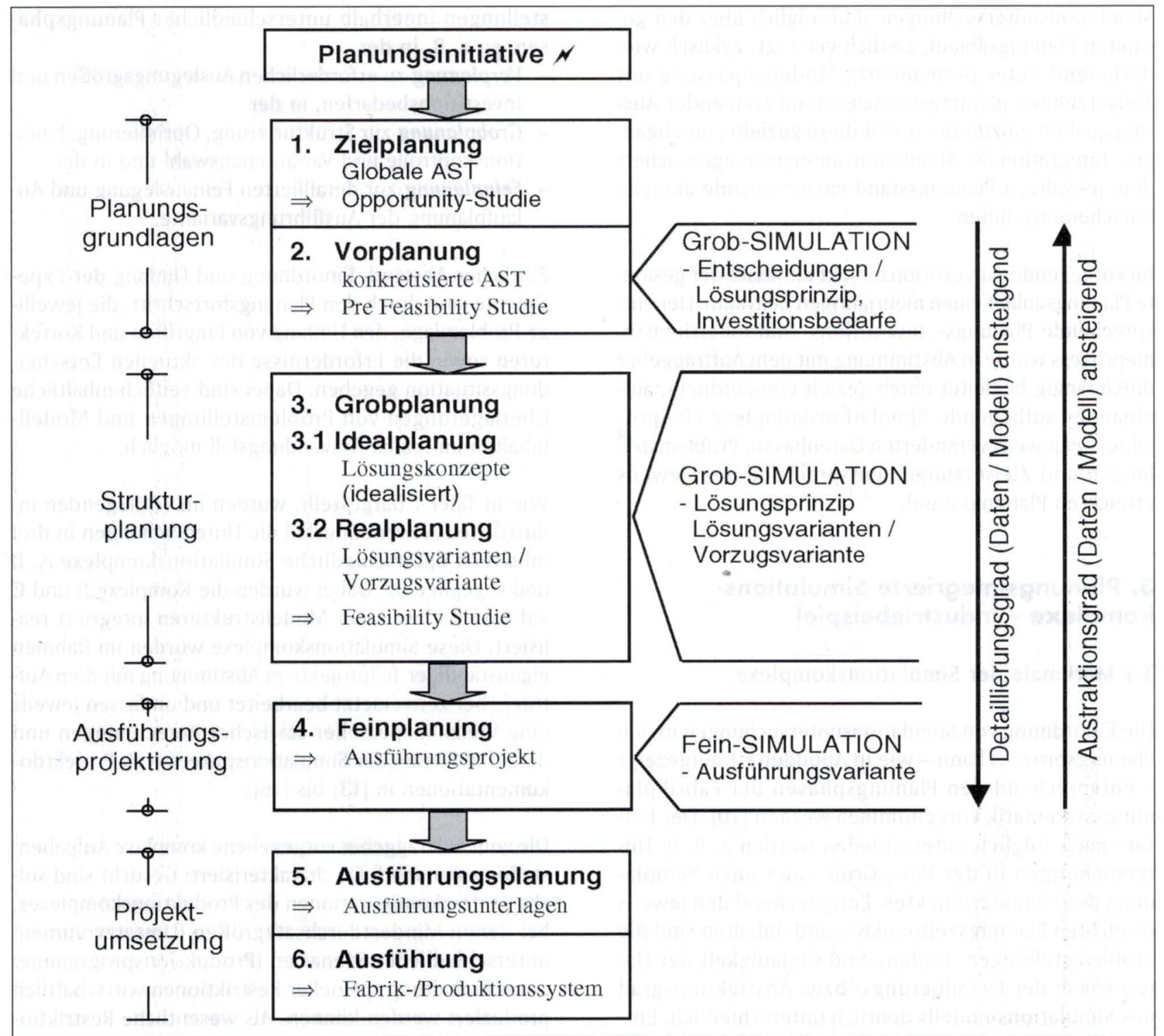

Abb. 1: Einordnung von Simulationsuntersuchungen in die Planungssystematik von Fabrik- und Produktionssystemen (i. A. an [10])

Bei der Konzeption der Simulationskomplexe war grundsätzlich davon auszugehen, dass Qualität und Umfang der Daten sowie die Vorstellungen zum Planungsobjekt einer ständigen Weiterentwicklung unterliegen. Insbesondere durch die parallel zum Planungsprozess ablaufenden evolutionären Prozesse der Produktweiterentwicklung, Marktaufklärung, Technologie-, Ausrüstungs- und Lieferantenspezifizierung sowie Logistikkonzeption war eine hohe Dynamik in der Modellgestaltung gegeben. Diesen Entwicklungen von Produkt- und Prozessaufklärung folgend wurde die angeführte globale Aufgabenstellung unter Zuordnung zu speziellen Planungsphasen der Planungssystematik - wie in Tafel 1 dargestellt - präzisiert:

- Teilprojekte - Simulationskomplex A

Szenarien (Produktionsprogramme) sind vorgegeben gesucht sind wirtschaftliche Systemgrößen und -strukturen als Basis für Investitionsentscheide - Prozessstufe Vorfertigung (VF)

- Teilprojekte - Simulationskomplexe B, C

Entsprechend dem Planungsfortschritt waren Teile der Ausrüstungsinvestitionen in der Realisierung, d. h. Systemstrukturen waren begrenzt vorgegeben.
Zielsetzungen waren nun die Überprüfung und die Korrektur bzw. Feinauslegung von Systemstrukturen auf der Basis erweiterter und aktualisierter Datenvorgaben - dabei gegliedert auf unterschiedliche Planzeiträume (Systemauslegung) sowie zeiteingegrenzt auf Anlaufszenarien (Inbetriebnahmephase), so dass inhaltliche Überlagerungen von Grob- und Feinplanung gegeben waren, die eine integrierte Bearbeitung der Simulationskomplexe B und C erforderten (vgl. Tafel 1). Diese Untersuchungen waren für die Prozessstufe „Vorfertigung (VF)“ sowie auf den Gesamtprozess erweitert für die zweite Prozessstufe „Fügeprozess (FP)“ durchzufuihren.

\subsection{Simulationskomplex A}

Prozessstufe: Vorfertigung (VF)

\section{Zielsetzungen}

- Bestimmung von Systemgrößen (Ausrüstungsbedarfe) auf Basis vorgegebener Szenarien (Produktionsprogrammstrukturen) 
- Ableitung von Investitionsumfängen

- Ableitung von Flächen- bzw. Raumbedarfen

\section{Restriktionen}

- Sicherung von Durchsatzgrößen (Umsatzvorgaben)

- Gewährleistung von Auslastungskenngrößen der Ausrüstungen

- Begrenzung und Homogenisierung von Staubildungen (Puffer- und Lagersysteme)

\section{Datenbasis}

- Grobanalysen zur mittelfertigen Markt- und Absatzentwicklung, daraus wurden Szenarien abgeleitet sowie Wachstums- und Entwicklungsetappen festgelegt

- Konstruktionsunterlagen Produkte (Entwicklungsund Funktionsmuster)

- Fertigungstechnologie (Verfahrenswahl, Ausrüstungsbestimmung), Grobvorgaben zu Arbeitsvorgängen, Rüst- und Bearbeitungszeiten in Abstimmung mit potenziellen Ausrüstungslieferern

- Vereinfachende Prozessvorgaben:

- Einschleusreihenfolgen - schematisiert

- Transportzeiten konstant

- Warteschlangenabbau

- Vorpuffer: FCFS-Regel

- Nachpuffer: Sofortabtransport

- Störungen ausgeklammert

- Losbezogener Transport (palettiert)

- Läger und Puffer ohne Kapazitätsbegrenzung

\section{Simulationssystem}

Fabrikplanungssystem MOSYS [11]

\section{Modellbildungsaufwand}

- Entwurf und Abgleich von Szenarien (Produktmix, Stuickzahlbereiche, Losreihenfolgen)

- Aufbereitung Arbeitspläne (Technologieabschätzung)

- Ableitung Operationsfolgediagramm und Funktionsschema

- Bestimmung von Losgrößen, Losanzahlen, Abgleich der Losgrößen mit Behältersystemen

- Berechnung von Basissystemen (statische Dimensionierung)

Deutlich werden die im Vorfeld der Simulationsuntersuchungen erforderlichen beträchtlichen Aufwände zur Bestimmung systemgerechter Datenstrukturen.

Der in der Planungsphase „Vorplanung“ den Untersuchungen zu Grunde gelegte Teilprozess (VF) war verfahrensund ausrüstungsbedingt durch 8 technologisch unterschiedliche Ausrüstungsarten (A1 bis A8) charakterisiert. Wie im Funktionsschema in Abbildung 2 dargestellt, dekken diese den Verfahrensbedarf breiter Produktsortimente (Szenarien) unterschiedlicher Technologie ab.

Erkennbar ist im Funktionsschema das Vorliegen eines einheitlich gerichteten Materialflusses interner Pro-

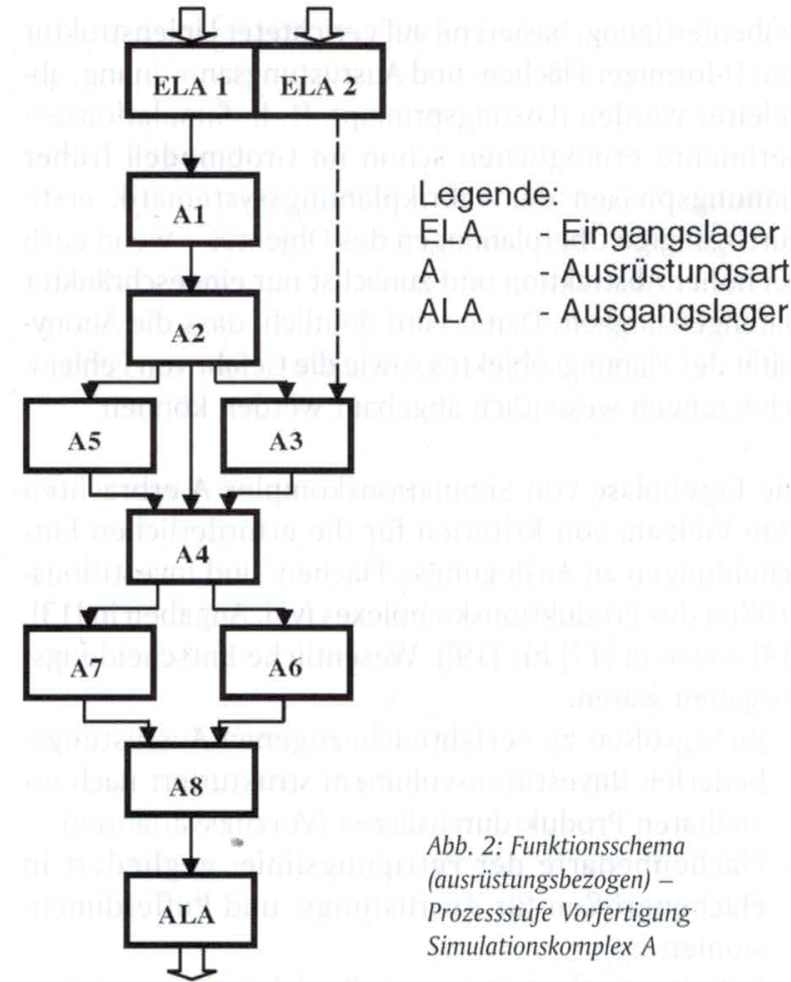

zessketten, aber auch Verzweigungen und Zusammenführungen treten auf. In Verbindung mit der Varianz von Einschleus- und Technologieparametern ist damit eine hohe Dynamik bzw. Turbulenz der Warteschlangenbildung der Produkte (Staueffekte) und der Ausrüstungen (Stillstände) zwingend zu erwarten. Damit wird auch an diesem „Kleinsystem“ deutlich, dass empirische Abschätzungen oder rein analytische Berechnungen zur Systemauslegung (z. B. bei Einsatz von Methoden der Bedienungstheorie) auf Grund der schon bei dieser Systemgröße im Praxisfall gegebenen Komplexität von Einfluss- und Verhaltensgrößen ausscheiden müssen. Die Notwendigkeit der Systemaufklarung durch experimentelle Untersuchungen bei Einsatz von Simulationstechniken ist damit auch unter diesen Bedingungen gegeben.

\section{Simulationsergebnisse}

Im Ergebnis der realisierten Simulationsexperimente wurden eine Vielzahl spezieller Kenngrößenuibersichten erstellt. Dadurch wurde schon in diesem frühen Planungsstadium eine hohe Transparenz sowie die Quantifizierung wesentlicher Parameter des Produktionskomplexes ermöglicht.

Zur Verdeutlichung dieser Ergebnisse sind in Tafel 2 szenarienbezogene Kennwerteübersichten ausschnittsweise angeführt. Diese ermöglichen vergleichende und alternative Analysen und Bewertungen zu erforderlichen Ausrüstungs-, Flächen- und Investitionsbedarfen. Auch sind Grenzbereiche wirtschaftlicher Nutzung bzw. Ausbaustufen (Investitionsetappen) des Produktionskomplexes ableitbar.

Von diesen Datenstrukturen ausgehend wurden die in Abbildung 3 dargestellten ,ersten“ Strukturentwürfe des Produktionskomplexes erstellt. Bei Beachtung vorgegebener Grenzgrößen der verfügbaren Flächen- und Raumstrukturen konnten als Lösungsprinzip die Fertigungsform 
Reihenfertigung, basierend auf gerichteter Linienstruktur und U-förmiger Flächen- und Ausrüstungsanordnung, abgeleitet werden (Lösungsprinzip). D. h. Simulationsexperimente ermöglichen schon im Grobmodell früher Planungsphasen der Fabrikplanungssystematik erste durchgängige Überplanungen des Objektes - wenn auch bei hoher Abstraktion und zunächst nur eingeschränkter Datengenauigkeit. Damit wird deutlich, dass die Anonymität des Planungsobjektes sowie die Gefahr von Fehleinschätzungen wesentlich abgebaut werden können.

Die Ergebnisse von Simulationskomplex A erbrachten eine Vielzahl von Kriterien für die erforderlichen Entscheidungen zu Auslegungs-, Flächen- und Investitionsgrößen des Produktionskomplexes (vgl. Angaben in [13], [14] sowie in [17] bis [19]). Wesentliche Entscheidungsvorgaben waren:

- Richtgrößen zu verfahrensbezogenen Ausrüstungsbedarfen (Investitionsvolumen) strukturiert nach erzielbaren Produktdurchsätzen (Vorzugsvarianten)

- Flächenbedarfe der Fertigungslinie, gegliedert in Flächengrößen für Ausrüstungs- und Pufferdimensionierung

- Kriterien zur Generierung von Produktionsprogrammen

- Eingrenzung wirtschaftlich realisierbarer Produktionsprogramme bzw. Ausruistungssysteme, Ableitung von Ausbaustufen (Investitionsetappen)

- Filterung von kapazitätssensiblen Ausrüstungs- und Pufferelementen (Sicherung der Erweiterungsfähigkeit durch gezielte Flächenvorhaltung)

- Grobentwüirfe von Anordnungsstrukturen (Lösungsprinzipien) des Produktionskomplexes

\subsection{Simulationskomplexe B, C}

Prozessstufen: Vorfertigung (VF) und Fügeprozess (FP)

\section{Zielsetzungen}

- Planungssicherheit bei der weiteren Auslegung und Inbetriebnahme der Produktionskomplexe

- Aktuelle Modifizierung des Gesamtkomplexes in die Prozessstufen Vorfertigung (VF) und Fügeprozess (FP)

- Für variierende aktuelle Produktionsstückzahlen war für beide Prozessstufen zu prïfen,

- ob die geplanten Kapazitäten der Maschinen und Anlagen der Vorfertigung noch real ausgelegt sind und in welchen Schichtstrukturen sie zu betreiben sind,

- wie hoch der Personalbedarf ist,

- wie die Lager- und Puffersysteme zu dimensionieren sind (Feinauslegung),

- ob bei termingerechter Bereitstellung der Einsatzteile im Zwischenlager (nach Fertigung in der mechanischen Vorfertigung) die vorhandenen Kapazitäten der Maschinen und Anlagen des Fügekomplexes ausreichend sind.

- Bereitstellung von Informationen zur Logistik der Auswärtsbearbeitung (Lohnfertigung) im Ablauf des Fügeprozesses

- Ableitung von notwendigen Folgeinvestitionen

\section{Restriktionen}

- Sicherung von Durchsatzgrößen nach aktuell modifizierten Umsatzvorgaben

- Gewährleistung von Auslastungskenngrößen der Ausrüstungen beider Teilprozesse

- Analyse und Homogenisierung der Warteschlangenbildung (Puffer- und Lagersysteme)

- Prozessgerechte Teilebearbeitung in der zweiten Prozessstufe (Fügeprozess) einschließlich der Auswärtsbearbeitung

\section{Datenbasis}

- Detaillierte Angaben (geplante Jahres- bzw. Monatsstuickzahlen) zu Produktionsprogrammen, von denen Szenarien abgeleitet wurden

- Erzeugnisstrukturen und Stuicklisten der Enderzeugnisse

- Reale Ausrüstungsstrukturen (Maschinen und Anlagen waren in Realisierung bzw. standen vor ihrer Installation)

- Feststehende Arbeitsvorgangsfolgen (Technologie) mit Auswärtsbearbeitung (Lohnfertigung) im Fügeprozess

- Vorgabezeiten lagen grob vor und wurden im Laufe des Teilprojektes durch Versuche in der Werkstatt präzisiert

- Transportzeiten wurden vernachlässigt

- Ausrüstungsausfälle (Störungen-Downtimes) wurden abgeschätzt und im Simulationsmodell berücksichtigt

\section{Simulationssystem}

Planungs- und Strukturierungssystem Pro Model [12]

\section{Modellbildungsaufwand}

\section{a) Vorarbeiten}

Um das nun deutlich modifizierte Simulationsmodell zu erstellen, waren neben Daten zum Produktionssystem (Maschinen, Lager, Fördermittel) Daten zu den Produktionsprogrammen (Produktsortimente, Losgrößen, Losanzahlen) und zu den aktuellen Bearbeitungstechnologien erforderlich. Dazu wurden die nachfolgenden Vorarbeiten für die Produktionskomplexe beider Produktionsstufen realisiert:

- Zusammenstellung der Sortimente an Enderzeugnissen und Einzelteilen entsprechend vorgegebener Unterlagen und Entwurf von Szenarien bezogen auf geplante Stuickzahlstrukturen (Programmvarianten)

- Bestimmung der Rohteilkosten

- Festlegung von Maschinengruppen und Maschinenstundenkosten

- Erstellung der Arbeitspläne (Überarbeitungen)

- Erstellung des Operationsfolgediagramms und des Funktionsschemas (VF, FP)

- Ermittlung von Losgrößen (zeitminimale, wirtschaftliche)

- Festlegung von Fertigungsauftragsgrößen entsprechend den ermittelten Losgrößen in Abstimmung mit dem Auftraggeber 


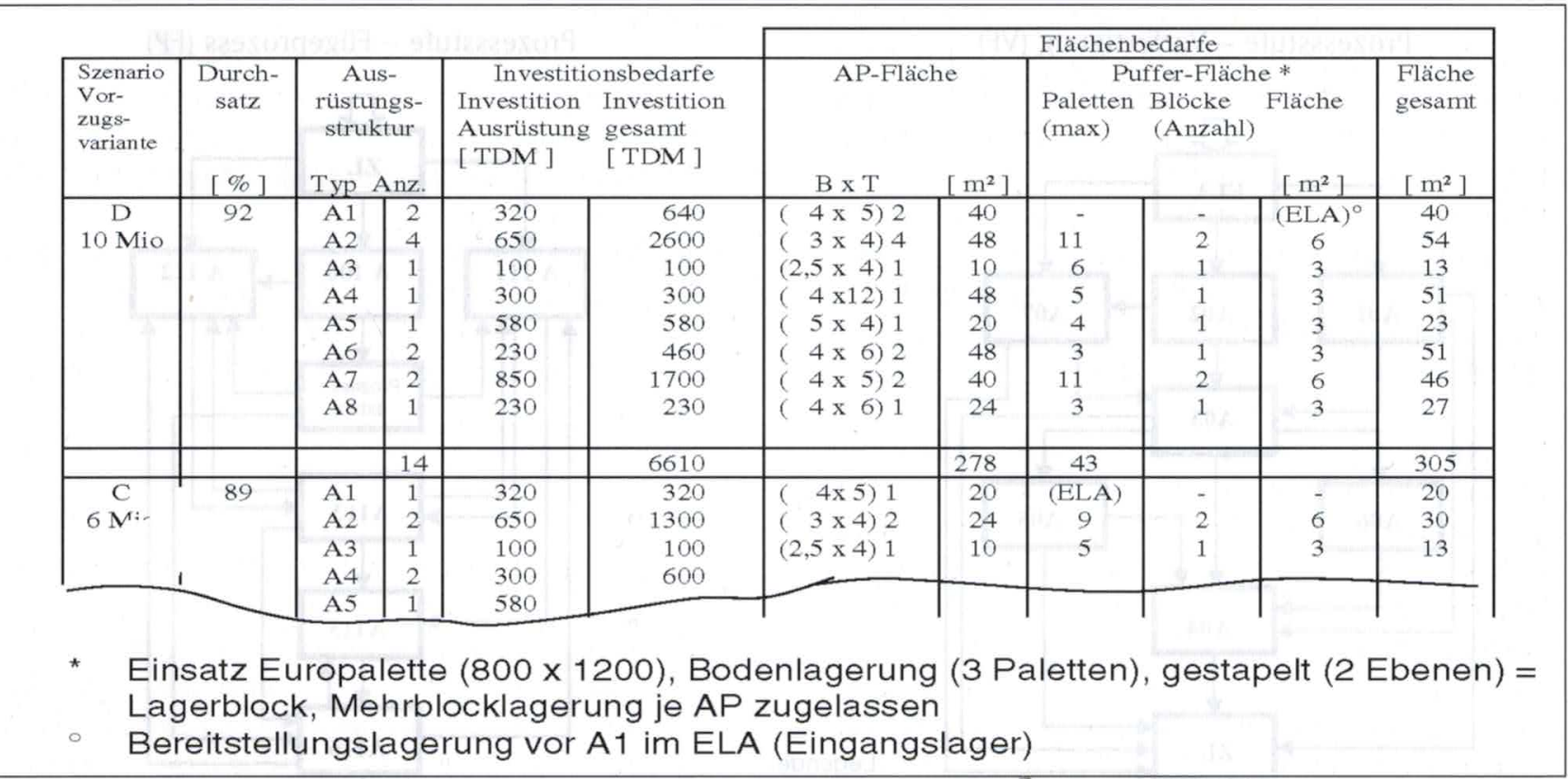

Tafel 2: Kennwerteübersichten - Ausrüstungs-, Investitions- und Flächenbedarfe des Produktionskomplexes (Varianten) [19]

\section{b) statische Berechnungen}

Als eine Voraussetzung füir die Versuchsdurchfuihrung (Experimente) wurden szenarienbezogene Basissysteme (statische Dimensionierung) berechnet. Diese bildeten die Experimentierplattform, auf die die Simulationsexperimente aufgesetzt wurden.

\section{c) Modellerstellung}

Entsprechend der Modifizierung bzw. Erweiterung des Produktionskomplexes in zwei Prozessstufen wurden autonome Simulationsmodelle für die Abbildung der Prozessstufe VF und der Prozessstufe FP entwickelt. Die Funktionsschemata beider Teilprozesse sind in Abbildung 4 dargestellt. Auf dieser Grundlage wurden unter Anwendung der Modellbildungsprinzipien des Simula- tionssystems ProModel die Teilprozesse im Simulator abgebildet (vgl. Abb. 5 und 6). Wesentliche Inhalte im Ablauf der Modellbildung sind in [15], [16] dokumentiert, wobei die technologische Aufarbeitung der Daten (Vorarbeiten) den größten Aufwand erforderte.

\section{Simulationsergebnisse}

\section{Prozessstufe - Vorfertigung (VF)}

Die Problemstellung bestand darin, die in der Vorplanung konzipierten Systemlösungen nun auf Basis deutlich aktualisierter Produktionsprogrammstrukturen (Szenarien) zu überprüfen bzw. erforderliche Korrekturen vorzunehmen. Auf Grund des fortgeschrittenen Planungs- bzw. teilweise eingeleiteten Realisierungsstan-

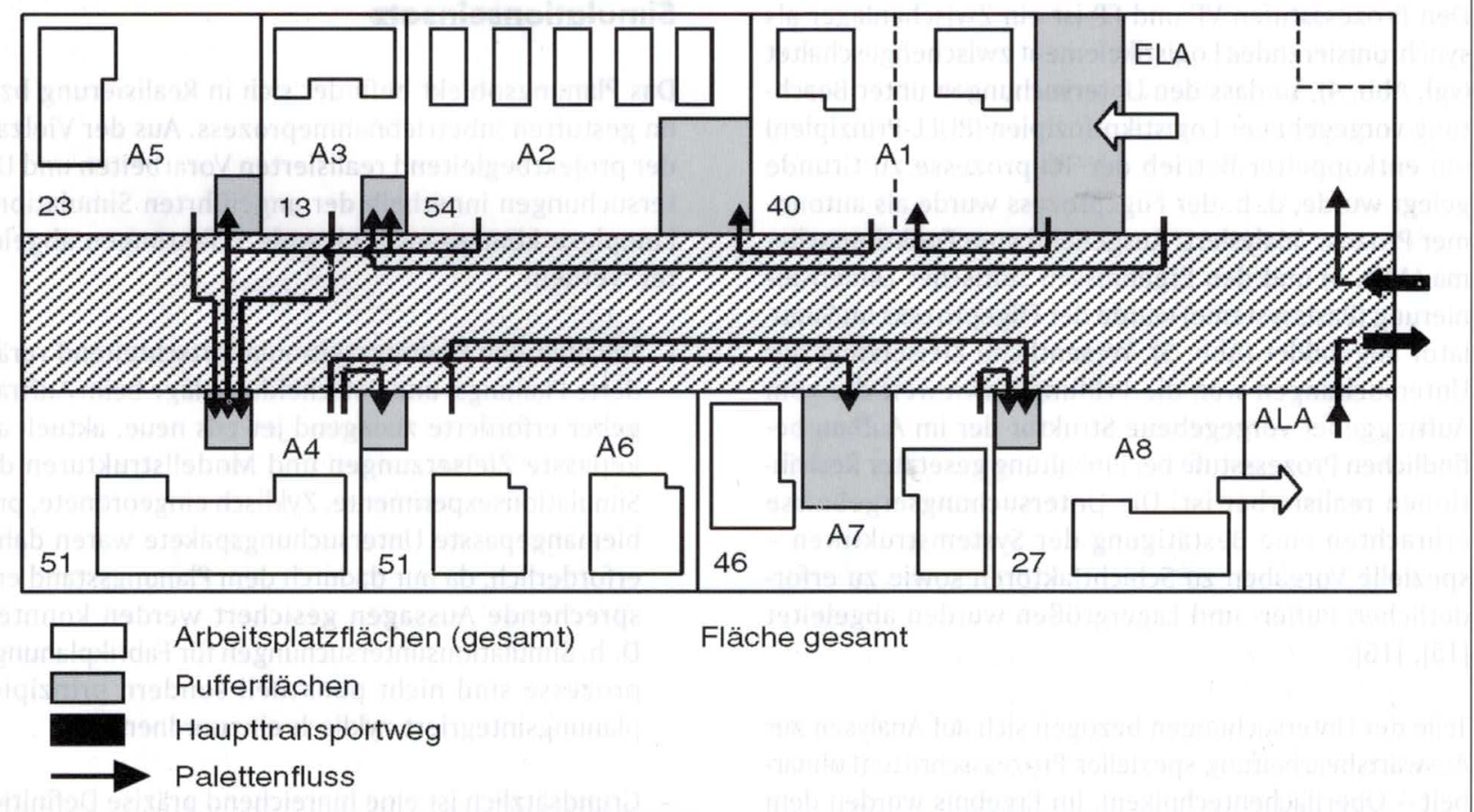


Prozessstufe - Vorfertigung (VF)

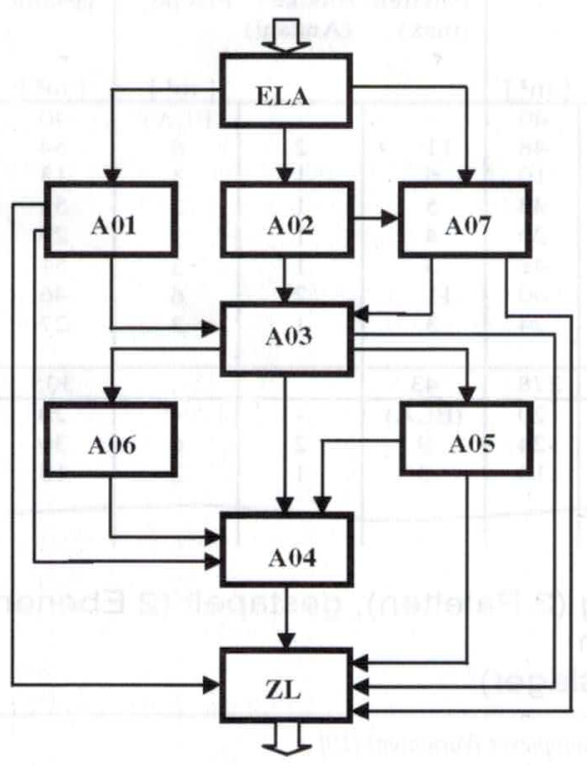

Prozessstufe - Fügeprozess (FP)

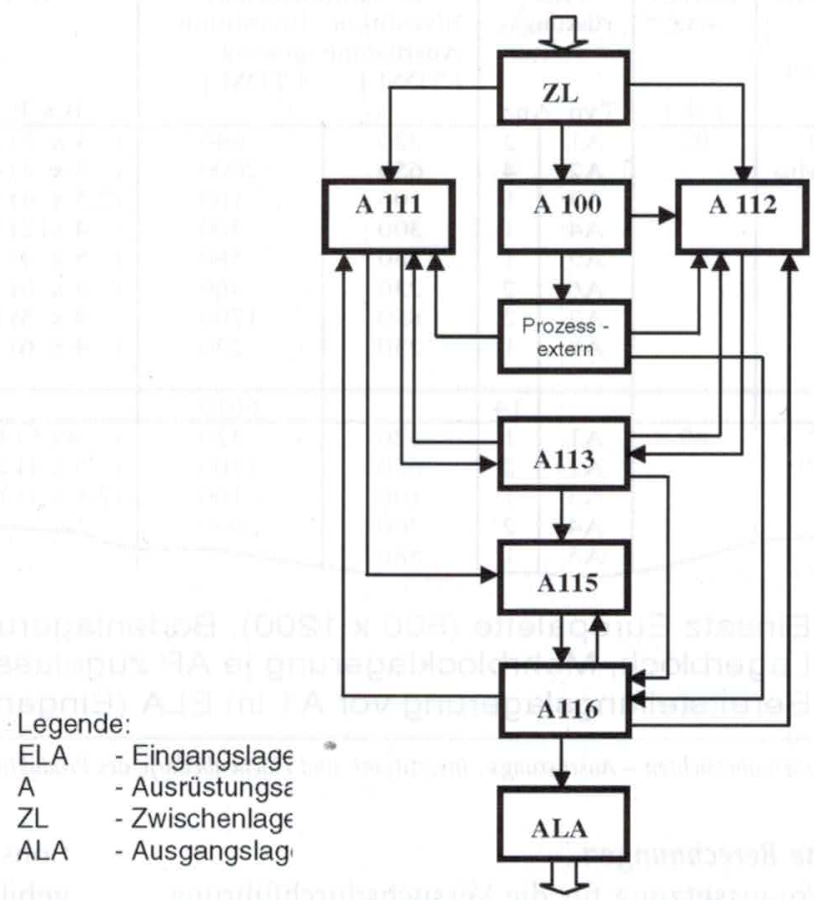

Abb. 4: Funktionsschemata (ausrüstungsbezogen) - Simulationskomplexe B, C

des bezogen sich die Korrekturen insbesondere auf die Anpassung von Schichtfaktoren, Puffer- und Lagergrößen (Bestandsgrößen) sowie auf die Auslastungshomogenisierung bei Sicherung aktueller Durchsatzgrößen. Im Ergebnis der Experimente wurden umfangreiche Statistiken (General Report) erstellt, aus denen Vorgaben für die Gestaltung des kurz- und mittelfristigen Systemablaufes abgeleitet wurden [15], [16]. Eine besondere Bedeutung kam dabei der Überprüfung spezieller Szenarien der Anlaufphase zu (Inbetriebnahmeprozess).

\section{Prozessstufe - Fügeprozess (FP)}

Den Prozessstufen VF und FP ist ein Zwischenlager als synchronisierendes Logistikelement zwischengeschaltet (vgl. Abb. 4), so dass den Untersuchungen unter Beachtung vorgegebener Logistikprinzipien (PULL-Prinzipien) ein entkoppelter Betrieb der Teilprozesse zu Grunde gelegt wurde, d. h. der Fügeprozess wurde als autonomer Prozess analysiert. Ausgehend vom Funktionsschema (Abb. 4) und den Ergebnissen statischer Dimensionierung (Basissysteme) wurde der Fügeprozess im Simulator abgebildet (Abb. 5). Wesentliche Zielsetzung der Untersuchungen war, die Prüfung, inwieweit die vom Auftraggeber vorgegebene Struktur der im Aufbau befindlichen Prozessstufe bei Einhaltung gesetzter Restriktionen realisierbar ist. Die Untersuchungsergebnisse erbrachten eine Bestätigung der Systemstrukturen spezielle Vorgaben zu Schichtfaktoren sowie zu erforderlichen Puffer- und Lagergrößen wurden abgeleitet [15], [16].

Teile der Untersuchungen bezogen sich auf Analysen zur Auswärtsbearbeitung spezieller Prozessschritte (Lohnarbeit - Oberflächentechniken). Im Ergebnis wurden dem Auftraggeber Informationen zu erforderlichen Transport- zyklen (z. B. Auftragsblockungen) erstellt, so dass Kriterien zur externen Transportlogistik erkennbar wurden.

Insgesamt zeigte sich, dass der Fügeprozess in den konzipierten Strukturen realisierbar ist und die Warteschlangenbildung eine nur begrenzte Turbulenz aufweist. Weitergehende Analysen deutlich veränderter Szenarien zeigten allerdings Kapazitätsengpässe bzw. Investitionserfordernisse auf, so dass mittelfristig erforderliche Entscheidungen sichtbar wurden.

\section{Erfahrungen - planungsintegrierter Simulationseinsatz}

Das Planungsobjekt befindet sich in Realisierung bzw. im gestuften Inbetriebnahmeprozess. Aus der Vielzahl der projektbegleitend realisierten Vorarbeiten und Untersuchungen innerhalb der angefuihrten Simulationskomplexe können nachfolgende Erfahrungen abgeleitet werden:

- Die über den Planungszeitraum fortschreitend veränderte Planungs- und Entscheidungslage beim Auftraggeber erforderte zwingend jeweils neue, aktuell angepasste Zielsetzungen und Modellstrukturen der Simulationsexperimente. Zyklisch eingeordnete, problemangepasste Untersuchungspakete waren daher erforderlich, da nur dadurch dem Planungsstand entsprechende Aussagen gesichert werden konnten. D. h. Simulationsuntersuchungen für Fabrikplanungsprozesse sind nicht punktuell sondern prinzipiell planungsintegriert zyklisch einzuordnen.

- Grundsätzlich ist eine hinreichend präzise Definition von Produktionsprogrammstrukturen (Szenarien) im 


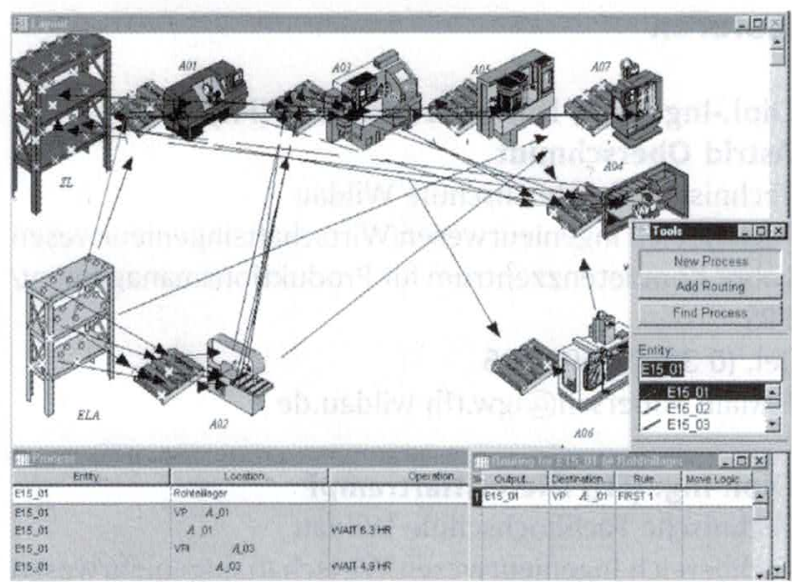

Abb. 5: Modellstruktur Prozessstufe - Vorfertigung (ProModel)

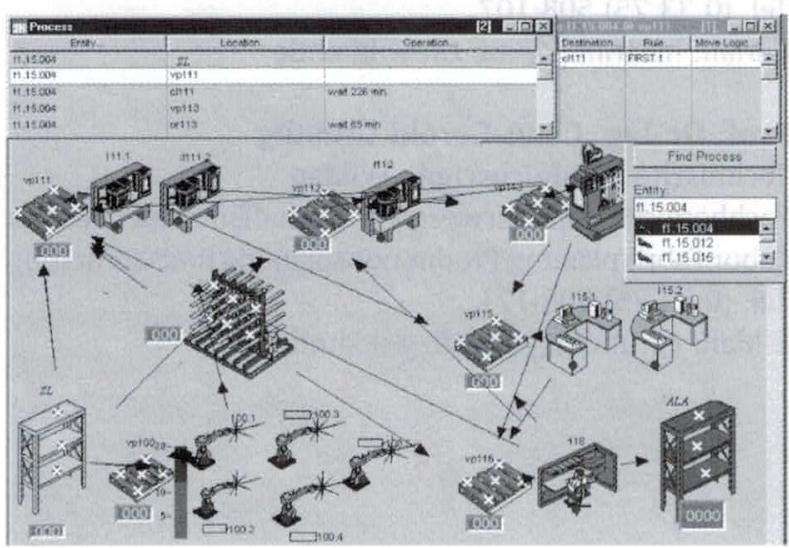

Abb. 6: Modellstruktur Prozessstufe - Fügeprozess (ProModel)

Ergebnis zu erwartender kurz- und mittelfristiger Markt- bzw. Vertriebsentwicklungen wesentlich problematischer als die Vorgabe von Entwürfen zu erforderlichen Verfahrens- bzw. Ausrüstungsstrukturen (vgl. auch [14], [17]). Gerade unter dieser Problemlage erweist sich die Simulationstechnik als ideales Analyseinstrument, da eine große Breite gezielt konzipierter alternativer Szenarien hochrationell getestet werden kann. Dabei sind auch optimistische und pessimistische Bereiche erfassbar, so dass ein breiter Raum des Systemverhaltens erkennbar wird.

- Eine abgestimmte Zusammenarbeit von Auftraggeber und Auftragnehmer zeigte sich als zwingend erforderlich, insbesondere für

- die Formulierung planungsaktueller Problemstellungen,

- die Daten- und Modellgestaltung sowie für

- die Bewertung der Untersuchungsergebnisse.

Nur eine teamorientierte Zusammenarbeit sichert die Akzeptanz und das Einfließen der Simulationsergebnisse in die Entscheidungslagen des Auftraggebers. Daraus leiten sich zwingend auch Anforderungen an den Auftragnehmer ab, wie z. B. die Notwendigkeit einer detaillierten und entscheidungsorientierten Dokumentation der Untersuchungen (vgl. Projektberichte [13] bis [16]) u. a. auch zur Sicherung der Ergebnisreproduzierbarkeit.

- Simulationstechniken ermöglichen schon in einer sehr frühen Planungsphase relativ präzise Entwürfe zu möglichen Systemstrukturen (vgl. z. B. Abb. 3). Allerdings sind dafür zur Definition des Simulationsmodells Daten bzw. Prozessmerkmale gefordert, die im Regelfall zu diesem Planungszeitpunkt nicht präzise vorgegeben werden können, d. h. im Regelfall nur abschätzbar sind auf Basis von Erfahrungen und Annahmen. Daraus folgt, der Einsatz der Simulationstechnik zwingt zum frühzeitigen Entwurf des geplanten Systems, erzwingt daher Transparenz, deckt offene Probleme im Systementwurf auf und provoziert folglich Diskussionen zum Planungsobjekt. Simulationseinsatz wird damit zum Motor der Systemaufklärung und planungsfortschreitender Präzisierung des Untersuchungsobjektes.

- Grundsätzlich erforderten die Simulationskomplexe einen beträchtlichen Vorbereitungsaufwand zur Erstellung der erforderlichen Basisdaten. Diese Aufwände, insbesondere für

- den Entwurf von Produktionsprogrammen (Szenarien),

- die Aufbereitung technologischer Daten (Arbeitspläne) und

- die Definition technologisch-organisatorischer Datenkomplexe

stellten sich als deutlich umfangreicher dar als die Aufwände für die Simulationsuntersuchungen. Die Planungsrealität zeigte oftmals, dass der Auftraggeber sich in konzeptionellen Vorstellungen befand. Die Simulationsexperimente allerdings benötigen konkrete Datenpools.

- Sichtbar wurde im Ergebnis des gesamten Investitionsprozesses, dass den Ergebnissen der Simulationskomplexe in der Vorplanung eine wesentliche Bedeutung zukommt. Jene legten Systemstrukturen vorab fest und bildeten die Grundlage für Ausrüstungsinvestitionen sowie den erforderlichen Flächenfreizug. Folgeuntersuchungen im Planungsfortgang sind von diesen Planungsrealitäten überlagert, d. h. Experimentierinhalte haben jetzt überprüfenden bzw. korrigierenden Charakter oftmals verbunden mit der Variantenbildung von Systemstrukturen. Daraus folgt, den Grobmodellen früher Planungsphasen kommt eine besondere Entscheidungsrelevanz zu!

\section{Literatur}

[1] Reinhart, G.; Feldmann, K.: Simulation - Schlïsseltechnologie der Zukunft? Stand und Perspektiven, München: Herbert UTZ Verlag, 1997

[2] Kuhn, A.; Rabe, M.: Simulation in Produktion und Logistik. (Fallbeispielsammlung), Berlin: Springer-Verlag, 1998

[3] Harder, K.: Simulation: Pro und Contra. Fördern und Heben 15 (1995) 3, S. 145-147

[4] Jünemann, R: Logistiksysteme. In: Betriebshütte - Produktion und Management (Teil 2), Eversheim, W.; Schuh, G. (Hrsg.), Berlin, Heidelberg: Springer-Verlag, 1996

[5] Hellingrath, B.: Hemmnisse des Simulationseinsatzes in kleinen und mittleren Unternehmen und deren Überwindung. Vortrag 8. ASIM-Fachtagung, TU Berlin 1998 
[6] Wenzel, S. (Hrsg.): Frontiers in Simulation. ASIM-Fortschritte in der Simulationstechnik, Erlangen: GrunerDruck, 2000

[7] Wiendahl, H.-P.: Grundlagen der Fabrikplanung, In: Betriebshütte - Produktion und Management (Teil 2). Eversheim, W.; Schuh, G. (Hrsg.), Berlin, Heidelberg: Springer-Verlag, 1996

[8] Kettner, H.; Schmidt, J.; Greim, H.-R.: Leitfaden der systematischen Fabrikplanung. München, Wien: Carl-HanserVerlag, 1984

[9] Grundig, C.-G.; Ahrend, H.-W.: Neuansatz der Fabrikplanungssystematik marktflexibler Produktionskonzepte. Werkstattstechnik 89 (1999) 6, S. 299-304

[10] Grundig, C.-G.: Fabrikplanung - Planungssystematik, Methoden, Anwendungen (Lehrbuch). München, Wien: Carl-Hanser-Verlag, 2000

[11] Fabrikplanungssystem MOSYS - Systemsoftware/Systemunterlagen, Fraunhofer Institut für Produktionsanlagen und Konstruktionstechnik (IPK) Berlin, 1993

[12] Simulationssystem Pro Model - Systemsoftware/Systemunterlagen, Gesellschaft für Betriebsorganisation und Unternehmensplanung, (GBU) mbh, Böblingen, 1999

[13] Grundig, C.-G.; Guhl, A.: Simulationsgestützte Auslegungsplanung Fertigungskomplex (Projektbericht 1 - Vorplanung). TFH Wildau, FB I/WI, Labor Fabrikplanung, 1998

[14] Grundig, C.-G.; Guhl, A.: Simulationsgestïtzte Analyse Fertigungskomplex (Projektbericht 2 - Vorplanung). TFH Wildau, FB I/WI, Labor Fabrikplanung, 1999

[15] Hartrampf, D.: Simulationsexperimente Investitionsvorhaben Kleinteilefertigung. (Projektbericht - Grob-/Feinplanung) TFH Wildau, FB I/WI, Labor Fabrikplanung, 2000

[16] Hartrampf, D.: Projektdokumentation - Statische Berechnungen, Simulationsexperimente, Prozessstufe VF - Teile I, II, Prozessstufe FP - Teile I, II, TFH Wildau, FB I/WI, Labor Fabrikplanung, 2000

[17] Grundig, C.-G.; Guhl, A.: Auslegungsplanung eines Fertigungskomplexes in einem mittelständigen Unternehmen unter Einsatz der Simulationstechnik (Industrieprojekt). Wissenschaftliche Beiträge der TFH Wildau (1998) 2, S. 57-67

[18] Grundig, C.-G.: Simulationstechnik sichert Transparenz des Investitionsobjektes. Zeitschrift für wirtschaftliche Fertigung (ZwF), 94 (1999) 5, S. 273-277

[19] Grundig, C.-G.: Anwendung der Simulationstechnik in der Praxis von KMU (Fallbeispiel). Vortrag auf der 9. ASIMFachtagung - Simulation in Produktion und Logistik. Fraunhofer Institut für Produktionsanlagen und Konstruktionstechnik (IPK) TU Berlin, März 2000, In: The New Simulation in Production and Logistiks. Mertins, K.: Rabe, M. (Hrsg.), Tagungsband, S. 117-126

\section{Autoren}

Dipl.-Ing. (FH), Dipl.-Wirtsch.-Ing. (FH)

\section{Astrid Oberschmidt}

Technische Fachhochschule Wildau

Fachbereich Ingenieurwesen/Wirtschaftsingenieurwesen Labor Kompetenzzentrum für Produktionsmanagement/ PPS

Tel. (0 33 75) 508-935

E-Mail: aobersch@igw.tfh-wildau.de

\section{Dipl.-Ing. (FH) Dieter Hartrampf}

Technische Fachhochschule Wildau

Fachbereich Ingenieurwesen/Wirtschaftsingenieurwesen Labor Fabrikplanung/Produktionsplanung und -steuerung Tel. (0 33 75) 508-107

E-Mail: hartramp@mb.tfh-wildau.de

\section{Prof. Dr.-Ing. Claus-Gerold Grundig}

Technische Fachhochschule Wildau

Fachbereich Ingenieurwesen/Wirtschaftsingenieurwesen Labor Fabrikplanung/Produktionsplanung und -steuerung Tel. (0 33 75) 508-171

E-Mail: cgrundig@igw.tfh-wildau.de 